\title{
El contexto epistemológico de la pedagogía crítica en el ajuste curricular del Ecuador en el 2016
}

\section{The epistemological context of critical pedagogy in the curricular adjustment of Ecuador in 2016}

Lcda. Zila Isabel Esteves Fajardo. Mgs.

Universidad de Guayaquil, Ecuador

Lcda. Norma Narcisa Garcés Garcés. Mgs.

Universidad de Guayaquil, Ecuador

Lcda. Sarita del Rocío Vizuete Flores. MSc.

Ministerio de Educación del Ecuador

Lcda. Patricia Estrella Acencio

Universidad de Guayaquil, Ecuador

Autor para correspondencia: zilaisabelesteves@ hotmail.es; norma.garcesg@gmail.com; sarita.vizuete@gmail.com; lidia.estrellaa@ug.edu.ec

Fecha de recepción: 02 de agosto de 2018 - Fecha de aceptación: 01 de diciembre de 2018

Resumen: Igual que las otras áreas, en el documento curricular, cada área se estructura en subniveles, organizando los aprendizajes en bloques curriculares que responden a criterios epistemológicos, didácticos y pedagógicos propios. Como la epistemología lleva el conocimiento desde el objeto del conocimiento, hacia el sujeto cognoscente, que por lo general es el estudiante, la forma cómo se acerque el conocimiento es lo pedagógico, y lo didáctico la forma que el docente intencionalmente acerca ese conocimiento a los demás. Desde esa óptica se elabora un documento para llegar a establecer los parámetros pedagógicos y didácticos que se realiza en el ajuste curricular.

Partiendo de cada criterio de evaluación, se describen los aprendizajes imprescindibles y deseables que los estudiantes tienen que alcanzar en cada área, se ofrecen orientaciones metodológicas y ejemplificaciones de tareas, y se especifican los objetivos generales del área a cuyo trabajo se contribuye analizando algunas áreas.

Palabras claves: ajuste curricular; epistemología; cognoscente; cognoscitivo

\begin{abstract}
Like the other areas, in the curricular document, each area is structure in sub-levels, organizing the learning in curricular blocks that respond own epistemological, didactic and pedagogical criteria. As epistemology brings knowledge from the object of knowledge, to the knowing subject, which is usually the student, the way is knowledge is approached is the pedagogical, and the didactic the teacher intentionally brings that knowledge to the rest. From this point of view, a document is prepared to arrive at establishing the pedagogical and didactic parameters the are carry out in the curricular adjustment.

Starting from each evaluation criterion, the essential and desirable learning that students have to achieve in each area is described, methodological orientations and examples of tasks are offered and the general objectives of the area to whose work is contributed are analyzed, analyzing some areas. Key words: curricular adjustment; cognitive; cognitive; epistemology
\end{abstract}




\section{Introducción}

\section{La Comprensión Epistemológica Aplicada En Las Operaciones Mentales}

¿Cómo afecta a la práctica didáctica en la aplicación del ajuste curricular por la falta de dominio de la epistemología y su la aplicación de los procesos dentro del aula?

La epistemología es parte de la filosofía y tiene como objeto el estudio del conocimiento humano estando entre ellos el del científico, sin descartar las diferentes fuentes y dimensiones que se manejan a nivel del currículo ecuatoriano. La educación maneja entre sus postulados la trasmisión del conocimiento de diferentes formas, por lo que, al pasar de una asignatura a otra, generalmente cambia la escuela filosófica que se aplica.

Tanto para el nivel de Educación General Básica como para el de Bachillerato General Unificado, los estudiantes, para avanzar hacia el perfil de salida, deben desarrollar aprendizajes aplicando desde el pragmatismo, el cognoscitivismo, el realismo, el intuicionismo, además de aplicar en el reconocimiento de procesos, a la fenomenología. Los objetos no emiten juicios, por lo que no tienen responsabilidad, que sí la tiene el ser humano, y en la medida de lo posible la educación les da el toque de diferencia, y en ese efecto, la epistemología le da ideas claras para aplicar la inducción y la deducción, y otros procesos como la clasificación y la síntesis.

A esa capacidad de responsabilidad, muchos la asocian de acuerdo a la capacidad del crecimiento cerebral que se posee, así quien tenga mayor madurez cerebral, tendrá mayor experiencia y se asocian así responsabilidades.

\section{Problema}

¿Cómo afecta a la práctica didáctica en la aplicación del ajuste curricular por la falta de dominio de la epistemología y su la aplicación de los procesos dentro del aula?

Variable dependiente: La práctica didáctica en la aplicación del ajuste curricular y su aplicación de los procesos dentro del aula.

Variable independiente: Falta de dominio de la epistemología

\section{Objetivos}

Fomentar la comprensión epistemológica aplicando las operaciones mentales en forma correcta para la práctica didáctica en la aplicación del ajuste curricular

Caracterizar a cada disciplina según su fundamento epistemológico para operacionalizar las variables didácticas dentro del aula.

La operacionalización de las áreas.

El bloque curricular agrupa y secuencia aprendizajes que pueden abarcar desde el primer año de la Educación General Básica hasta el último del Bachillerato General Unificado, constituyéndose en una división longitudinal del área a lo largo de los estudios obligatorios. En 
esa división longitudinal se pone en práctica la dosificación o la segmentación del fondo de cada contenido. Pero además en la secuenciación se logra ir de menos a más, porque el conocimiento del ser humano no llega al 100\% dependiendo del nivel de estructura científica, natural o vulgar.

Se van a determinar cómo se aplican las diferentes escuelas filosóficas, desde las siguientes áreas de conocimiento:

Estudios Sociales. El área de Estudios Sociales le da también al estudiante la capacidad de desarrollar la axiología, la medición y la observación de las realidades. El estudiante deja de ser un simple lector de los contenidos curriculares para asumir unas competencias procedimentales en la crítica social de lo que observa, dentro de un ámbito positivista de la historia.

En el enfoque realista y no surrealista que debe tener el análisis de los derechos humanos, una de las competencias básicas es la observación social de los problemas de los problemas y el análisis causa efecto junto con la comparación de la normativa, descubriendo su desarrollo y con ello demostrar si tales derechos se cumplen o no.

Mediante esquemas causales o líneas de tiempo, así como mapas de evidencias los estudiantes pueden construir su propia historia en cualquier ámbito, pero deben partir de ciertas hipótesis. Así el ejercicio de elaboración de hipótesis en la historia ha permitido elaborar evidencias que llevan a demostrar la importancia de la organización lógica para hacer una buena historia.

Ya al narrar sobre la pedagogía de los derechos civiles y humanos (Vila, 2010) manifiesta que: "El carácter progresivo en la historia de la conformación y estructuración de los derechos humanos, no permite hacer síntesis sin acumular ni asumir lo ganado, por medio de luchas y conquistas sociales. La praxis histórica convierte la tradición de los derechos humanos en una forma cada vez más compleja y rica, no susceptible de mutilaciones; si no, no podríamos hablar de derechos humanos."

Pues tal como lo dice Vila que la praxis histórica es tan rica que no vale mutilarla, siendo la Educación para la Ciudadanía una forma de reconocer la evolución de la historia y la hipótesis una estrategia del nivel educativo de bachillerato con la finalidad de llegar a convertir al estudiante no solamente en lector, sino en un futuro ensayista comparando ideas de acuerdo a las necesidades que va a requerir su vida universitaria.

En la medida que los estudiantes lean la historia, califican a los personajes y mediante ese análisis llegan a una serie de conclusiones que se transforman bien formuladas y con sus respectivas premisas en hipótesis. ¿Estamos formando estudiantes críticos, dogmatizados por el currículo o porque la historia de los derechos humanos puede servir a interpretar los derechos en el siglo XXI en toda su conceptualización? Ya Cabaluz en la Revista Aletheia de la Universidad de la Plata, enfocaba la forma crítica de la pedagogía y sus ideas iban orientadas a asumir nuevas competencias en el bachillerato. Ya decía que:

Las pedagogías críticas emergentes en la década de los 60's, estuvieron en sus orígenes 
principalmente influenciadas por las teorías de las teorías de la reproducción, y enmarcaron los sistemas educativos en los procesos de dominación social. La educación legitimaba los beneficios de los grupos hegemónicos y contribuía a reproducir las relaciones sociales de explotación y opresión incorporando y desplegando en todos los espacios educativos una multiplicidad de mecanismos de control y disciplinamiento.

Me parece acertada la posición de Cabaluz, al invitar a la crítica en los espacios educativos, para que estos no sirvan como sistemas de opresión sino de conocimiento para llegar a la verdad.

Si bien muchas veces el estudio de Educación para la ciudadanía es una herramienta básica para que el estudiante pueda ser un conocedor de la norma, la historia le puede ayudar a alcanzar la competencia con el método hipotético para alcanzar valores sociales y axiológicos de enorme relevancia en el ejercicio de una ciudadanía responsable; porque lo que se busca es que el estudiante adquiera la competencia de indagador de la realidad nacional del Ecuador

Lengua y Literatura: es un área de conocimiento fundamentada en lo general por la teoría, de la misma sus contenidos la historia de la cultura los ha ido plasmando, de tal forma que, a partir del esfuerzo humanístico, se han ido formando sus contenidos.

Según Gardner (2012), sobre la inteligencia lingüística, nos menciona que: Es considerada una de las más importantes. En general se utilizan ambos hemisferios del cerebro y es la que caracteriza a los escritores. El uso amplio del lenguaje ha sido parte esencial para el desarrollo de este tipo de inteligencia.

Se transforma en pragmática en el siguiente análisis literario, por ejemplo, la docente da la siguiente instrucción: a partir del siguiente fragmento, seleccionen alguna figura literaria y la posición del adjetivo.

En las calles de Durán juegan dos niños a la pelota, con ese ánimo propio de aquellos que saben que es un sábado con poco sol y con muchas madres y abuelas que vienen y van desde los mercados de la zona. Dos árboles, uno de coco, una palma, y otro de pino, sirven de arco para Milton y Andrés.

El análisis es la operación mental que se utiliza para este proceso. En la lectura se descubre la presencia de una topografía al describir el lugar lleno de árboles. Pues luego de la operación del análisis está presente la síntesis. El análisis son los pasos progresivos y poco a poco que se debe utilizar en el descubrimiento de la topografía.

También se definen indicadores de evaluación que secuencian y concretan los estándares de aprendizaje y sirven para evaluar el logro progresivo del perfil de salida. 


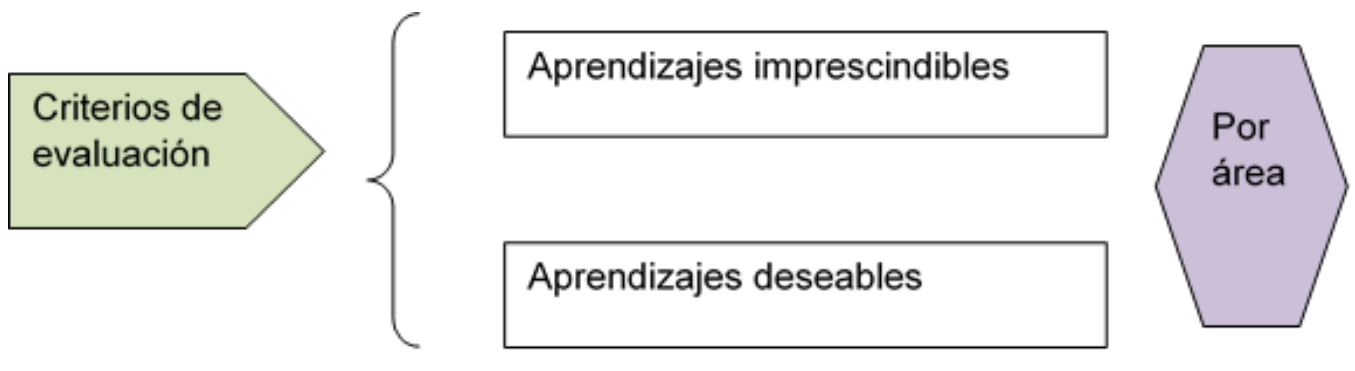

Figura 1

Por último, se ofrece un mapa de los contenidos conceptuales que se proponen para cada subnivel de la Educación General Básica y para el Bachillerato General Unificado, según el caso. Todos estos elementos, así como la forma en que se estructuran e interrelacionan en la propuesta, han sido pensados para facilitar el trabajo colaborativo de los docentes en torno al desarrollo de una propuesta curricular concreta para sus instituciones educativas.

El estudiante es un ser axiológico, mide y es medido, observa y es observado, se implanta en su capacidad cognoscente una serie de juicios valorativos que lo convierte al ser humano en el primer ser de la naturaleza que puede emitir juicios racionales con gran capacidad analítica.

\section{Ciencias Naturales}

Como a la epistemología le interesa analizar los criterios de verdad, la ciencia le proporciona los elementos de forma objetiva, de tal manera que se maneja bajo las categorías. Las categorías ya las estudió Enmanuel Kant, y Aristóteles también y se relacionan con: el color de los huesos, de los elementos celulares, de las hojas, con la clorofila. La categoría tiempo con todo lo que implique los procesos de mitosis y meiosis, y el mismo crecimiento muscular.

En los escritos de Karl Popper y Konrad Lorenz, que fueron los más destacados investigadores del comportamiento animal y que llegó a ocupar la más elevada posición filosófica en London School of economics and political science y reconocido entre los científicos de la conducta por sus aportes en la metodología de la investigación, la epistemología, ha aportado a la ciencia lo mejor en cuanto los procesos cognitivos y de razonamiento, que evita que se desvíe de su objetivo, llegar al utilitarismo hacia el bienestar de lo biológico y humano.

En la relación sujeto/objetos, está íntimamente relacionado con el conocimiento, el descubrimiento y el contexto de la realidad para ser categorizada, permitiendo delimitar al objeto, sin que se vea afectado el objeto, pero el sujeto del conocimiento hace suya esa realidad en base a la aprehensión como la que la hace frente a un microscopio.

El conjunto del contexto del descubrimiento de las diferentes realidades, muchas veces segmentadas, permite delimitar el objeto y ese procedimiento de análisis y reflexión, da la justificación lógica, sobre de donde surge el objeto para hacer posible la teoría del conocimiento, las teorías de las ciencias y los criterios expresados filosóficamente, los estudios científicos, filosóficos, matemáticos y lógicos, estudia las cualidades y las leyes del mundo. Se puede decir que existe una epistemología del derecho, de la ciencia, de la educación, no muy desarrolladas, pero tampoco no muy complicada de entender. 
Si se aplica el descubrimiento a la educación básica, sería bueno ejemplificar ésta etapa del conocimiento al ver, escuchar, tocar, oler, actos, la mayoría de ellos relacionados con la percepción. En ese momento se convierte el conocimiento en una labor que se auxilia de la metodología para adquirir el valor cognitivo. Ese valor cognitivo lleva a la formación de ideas del mundo real. Ese mundo interior del conocimiento hace que se generen los elementos que ayudarán luego al establecimiento del juicio.

La nueva propuesta curricular está estructurada por áreas y promueve el trabajo interdisciplinar. Cada una de las áreas orienta el trabajo del aula a partir de asignaturas que recogen las intenciones educativas y las organizan disciplinariamente para facilitar el trabajo docente, pero se debe subrayar la necesidad de generar situaciones de enseñanza y aprendizaje lo más integradoras y significativas posible, sobre todo en los primeros años de la educación obligatoria.

En el aprendizaje también se genera conocimiento. Pero ¿cuál es la diferencia entre epistemología y aprendizaje? Bien, en el aprendizaje se da la generación de conocimiento, pero en su mayoría muchos de esos aprendizajes no siguen las leyes de la epistemología para su desarrollo.

¿Qué sucede cuando el aprendizaje logra incorporar lo mejor de la epistemología? Pues sucederían muchísimas instancias de mejoramiento en la enseñanza de las ciencias y disciplinas, sobre todo en la metodología, porque lo que aporta la epistemología al aprendizaje es las formas cómo el mediador del conocimiento debe lograr que la zona de desarrollo próximo al estilo de Vygotsky le permita llegar a la aprehensión del objeto de estudio con un andamiaje didáctico valorado por docentes experimentados que aplican las formalidades pedagógicas según el tipo de inteligencia que el estudiante posea de acuerdo a la teoría de Gardner.

Concreción del área por subniveles. Incluye los elementos generales que definen, caracterizan y configuran el área en cada subnivel de la Educación General Básica y en el Bachillerato General Unificado: la contribución del subnivel a los objetivos generales del área, los objetivos específicos del área para el subnivel, los contenidos, expresados en destrezas con criterios de desempeño y estructurados en bloques disciplinares, y los criterios de evaluación, que presentan el desarrollo curricular del área.

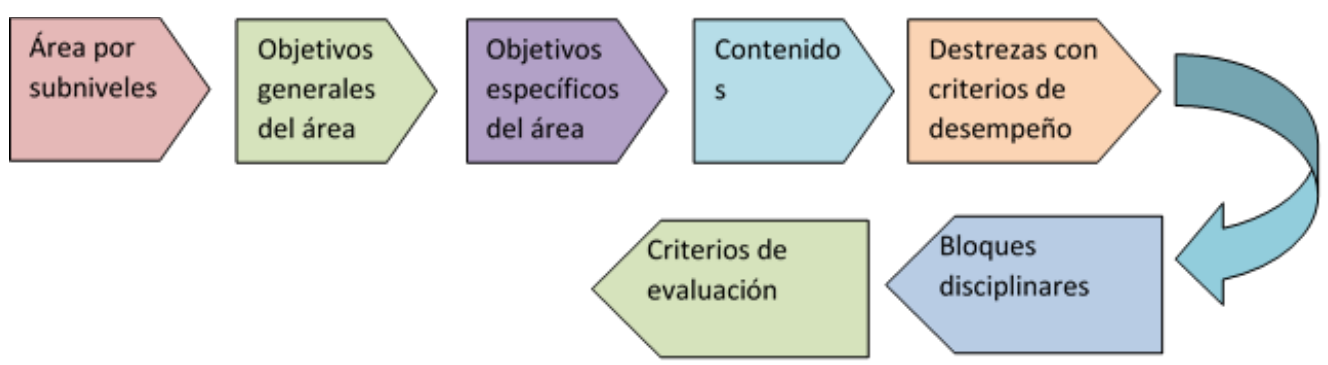

Figura 2 
Física. Según (Schwartz, 1987) Parte Popper de la reflexión de que, frente a las ideas materialistas clásicas, los físicos actuales han rechazado la idea de que el universo está determinado, de que no puede haber otra novedad en el universo, que una novedad de combinación de elementos preexistentes. La mecánica cuántica ha echado por la borda el determinismo estricto. Puede haber leyes invariantes y emergencia, ya que el sistema de leyes invariantes no es el complejo y restrictivo para evitar la emergencia de nuevas propiedades legales. (Pág. 155)

Con ello la ciencia se vale de la epistemología en estos aspectos: calidad de la observación, el análisis, la separación aristotélica o kantiana de las categorías. Así cuando la ciencia quiere aplicar los métodos de la teoría del conocimiento, se auxilia de la filosofía y se convierte en la filosofía de la ciencia, en epistemología de la ciencia y se preocupará de explicar cómo la ciencia produce el conocimiento desde un punto de vista experimental y demostrativo, llegando para aquello a través de ciencias físicas, biológicas, matemáticas y otras, incluidas las tecnológicas.

Según Gardner (2012), sobre la inteligencia espacial, menciona que: Esta inteligencia la tienen las personas que puede hacer un modelo mental en tres dimensiones del mundo o en su defecto extraer un fragmento de él. Esta inteligencia la tienen profesiones tan diversas como la ingeniería, la cirugía, la escultura, la marina, la arquitectura, el diseño y la decoración. Por ejemplo, algunos científicos utilizaron bocetos y modelos para poder visualizar y decodificar la espiral de una molécula de ADN.

La inteligencia espacial es la habilidad para percibir de manera exacta el mundo visualespacial, incluye la sensibilidad al color, la línea, la forma, el espacio y las relaciones que existen entre estos elementos. Incluye la capacidad de visualizar, de representar de manera gráfica ideas visuales o espaciales.

\section{Metodología}

Se aplica una encuesta a diez docentes de la Escuela de Párvulos de la Universidad de Guayaquil. La investigación que se realizó está direccionada, por el método del constructivismo crítico y social, que puntualiza en el aprendizaje como un cambio de formas, tanto en el ambiente educativo como el entorno social, y para poder aprender resulta que la investigación se realizó con estudiantes del tercer semestre de la Escuela de la carrera de Educación de párvulos de la Universidad de Guayaquil. En este proceso de aprendizaje requiere organizar los estímulos en la mejor manera que los estudiantes pueden dar respuesta adecuadas y recibir mejor la retroalimentación, para poder establecer las mejores directrices para que los estudiantes puedan asimilar y aprender los contenidos que se desea alcanzar para que puedan dominar el tema de la epistemología. 


\section{Resultados}

\section{Encuesta para artículo indexado}

1.- El docente moderno debe ser de la escuela epistemológica realista para diferenciar entre su realidad interior y la realidad del mundo de su trabajo.

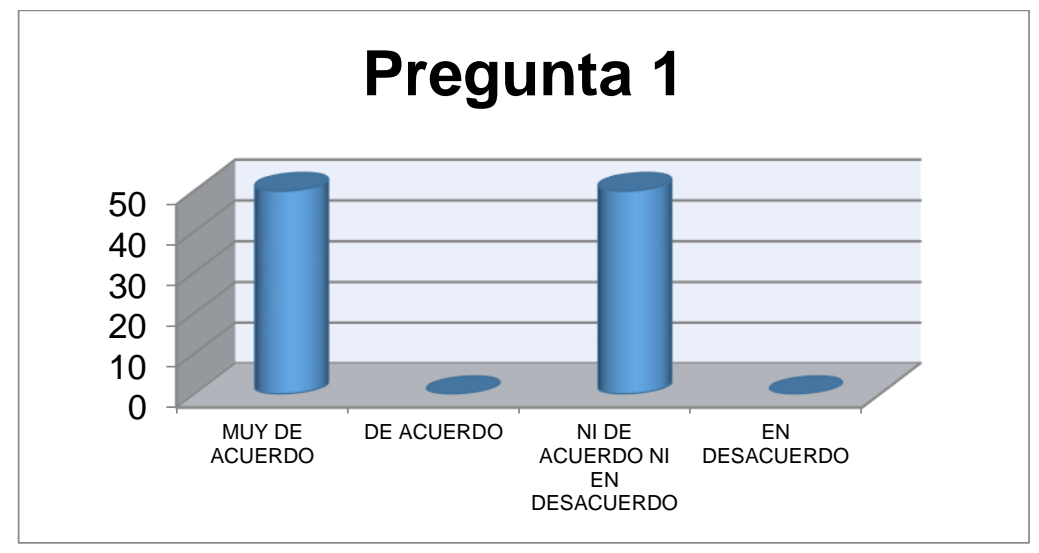

Figura 3

Las estudiantes consideran en un 50\% estar muy de acuerdo con que el docente moderno debe ser realista para de esa forma su realidad interior y la realidad del mundo del trabajo, las tome como elementos complementarios.

2.- Cuando no somos neorrealistas y no se ve alterada la sana crítica

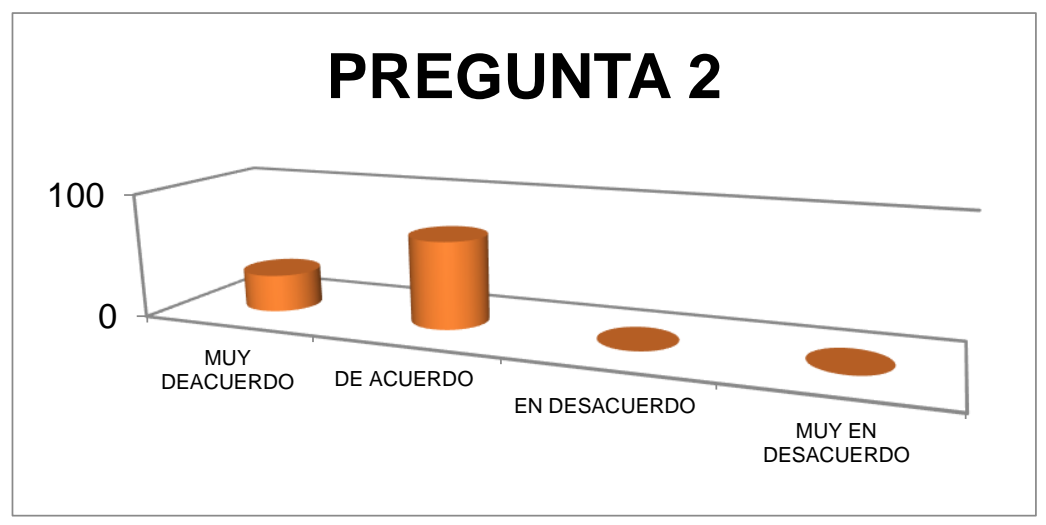

Figura 4

El 30\% están muy de acuerdo que la sana crítica acompañada del realismo no permite que se altere el juicio, y el $70 \%$ está de acuerdo con esa misma apreciación.

3.- Los elementos que benefician a la criticidad en el Área de Ciencias Naturales son el buen juicio y la objetividad 


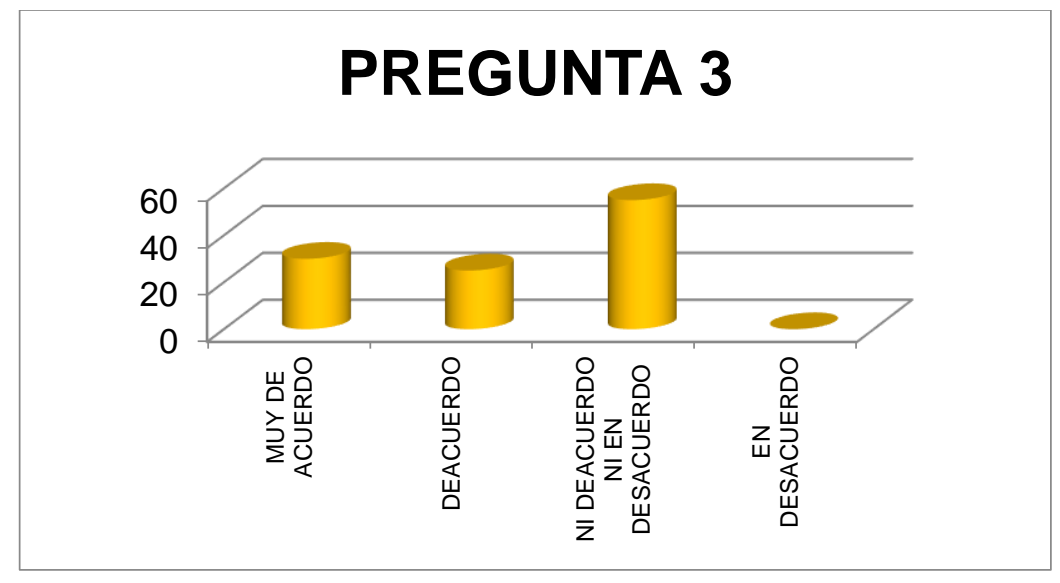

Figura 5

El 30\% están muy de acuerdo con que la criticidad se ve beneficiada por el buen juicio y la objetividad. El $25 \%$ está de acuerdo y el $55 \%$ es indiferente a esta realidad.

4.- De los cinco, uno de los siguientes elementos le pertenece al realismo crítico.

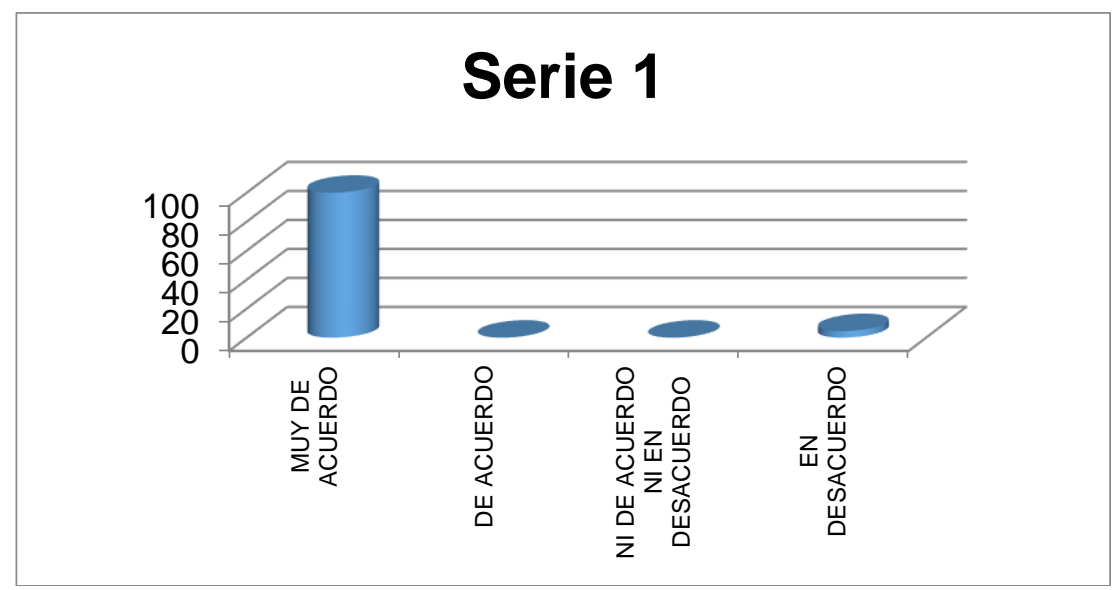

Figura 6

a) Muy de acuerdo con la realidad y analizarla objetivamente

b) De acuerdo con la realidad y no querer mejorarla

c) Indiferente ante la realidad

d) En desacuerdo con la realidad

e) Muy en desacuerdo con la realidad pero no quererla cambiar

El $100 \%$ considera que los elementos que pertenecen al realismo crítico son la realidad y analizarla objetivamente.

5.- Las nuevas corrientes de pensamiento necesitan de docentes profundamente analíticos y discursivos. 


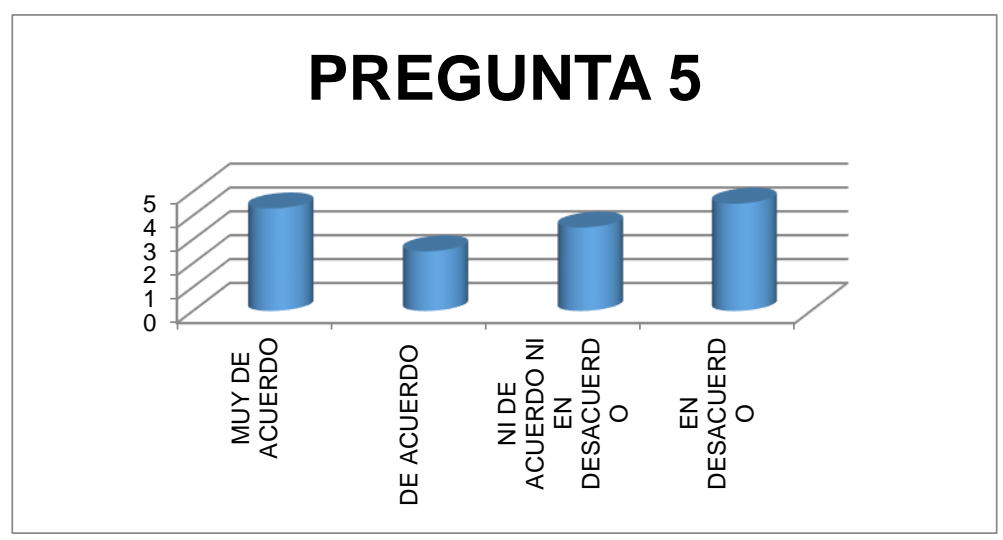

Figura 7

El 80\% de los estudiantes está muy de acuerdo con que las nuevas corrientes de pensamiento necesitan docentes profundamente analíticos y discursivos. El 20\% está de acuerdo con esta premisa.

\section{Conclusiones}

La política educativa, estudia aspectos subjetivos, políticos, éticos, sociales e ideológicos; apunta desde un carácter científico, someter a un criterio de confrontación, legitimación, frente a todo lo que está carente de sentido y lógica, acudiendo a la racionalidad científica, acorde a la situación que se vive en el mundo, la ciencia, la tecnología, el saber, el conocimiento.

Como los aprendizajes contenidos en cada uno de los bloques curriculares de las distintas áreas que conforman la educación obligatoria se ordenan en torno a los objetivos que en cada subnivel de la Educación General Básica marcan la secuencia para el logro de los objetivos generales del área al culminar el nivel de Bachillerato General Unificado, estos están expresados en términos de capacidades que se pretenden alcanzar y son el núcleo sobre el que se articulan todos los elementos del currículo.

Esta organización del currículo permite mayores grados de flexibilidad y apertura curricular y responde al objetivo de acercar la propuesta a los intereses y necesidades de los estudiantes, a la vez que permite que esta se adapte de mejor manera a sus diferentes ritmos de aprendizaje.

El reajuste del currículo ha sido diseñado mediante destrezas con criterios de desempeño que apuntan a que los estudiantes movilicen e integren los conocimientos, habilidades y actitudes propuestos en ellas en situaciones concretas, aplicando operaciones mentales complejas, con sustento en esquemas de conocimiento, con la finalidad de que sean capaces de realizar acciones adaptadas a esa situación y que, a su vez, puedan ser transferidas a acciones similares en contextos diversos.

De este modo, se da sentido a los aprendizajes, se establecen los fundamentos para aprendizajes ulteriores y se brinda a los estudiantes la oportunidad de ser más eficaces en la aplicación de los conocimientos adquiridos a actividades de su vida cotidiana. 


\section{Recomendaciones}

Es imprescindible tener en cuenta la necesidad de contextualizar los aprendizajes a través de la consideración de la vida cotidiana y de los recursos del medio cercano como un instrumento para relacionar la experiencia de los estudiantes con los aprendizajes escolares.

Los principios para el desarrollo del currículo que se acaban de enunciar han de incidir en las programaciones didácticas que elaboren las instituciones educativas para los niveles de educación obligatoria, considerando la atención a la diversidad y el acceso de todo el alumnado a la educación como principios fundamentales de esta tarea.

Se debe fomentar una metodología centrada en la actividad y participación de los estudiantes que favorezca el pensamiento racional y crítico, el trabajo individual y cooperativo del alumnado en el aula, que conlleve la lectura y la investigación, así como las diferentes posibilidades de expresión.

Este enfoque implica que el proceso de enseñanza y aprendizaje debe abordarse desde todas las áreas de conocimiento y por parte de las diversas instancias que conforman la comunidad educativa.

\section{Bibliografía}

Csikszentmihalyi, M. (Marzo de 2006). www.wikispaces.com. Obtenido de www.wikispaces.com:https://catedrabdcv.wikispaces.com/file/view/el+juego+y+la+creati vidad.pdf

Gardner, H. (19 de 12 de 2012). Transformando el infierno. Obtenido de los 8 tipos De inteligencia según howard gardner: la teoría de las inteligencias múltiples: https://transformandoelinfierno.com/2012/12/19/los-8-tipos-de-inteligencia-segunhoward-gardner-la-teoria-de-las-inteligencias-multiples/

Klimovsky, G. (30 de mayo de 2005). Ciencia, Tecnología y Sociedad. Obtenido de Epistemología y prácticas del conocimiento: http://www.revistacdyt.uner.edu.ar/articulos/descargas/cdt30_guyot.pdf

Lorenz, K. (1993). Hablaba con las bestias, los peces y los pájaros. Barcelona: Labor.

Muro, E. (2010). Elementos esenciales en la enseñanza de la Técnica Legislativa.

Nancy Montes de Oca Recio, E. F. (2011). Estrategias docentes y métodos de enseñanza aprendizaje en la Educación Superior. XI (3).

Piaget. (1984). Construcción real en el niño.

Schwartz, P. (1987). Karl Popper y la Teoría de la Evolución. Teorema XIV, 145. 
Vila, A. M. (2010). Ciudadanía, derechos sociales y educativos: Reflexiones para una pedagogía de los derechos humanos. Archivos analíticos de políticas educativas. 